\title{
\begin{tabular}{l|l} 
MitTraries & DSpace@MIT
\end{tabular}
}

\author{
MIT Open Access Articles
}

\section{Blood-brain-barrier organoids for investigating the permeability of CNS therapeutics}

The MIT Faculty has made this article openly available. Please share how this access benefits you. Your story matters.

Citation: Bergmann, Sonja et al. "Blood-brain-barrier organoids for investigating the permeability of CNS therapeutics." Nature Protocols 13, 12 (December 2018): 2827-2843 (c) 2018 Springer Nature

As Published: http://dx.doi.org/10.1038/s41596-018-0066-x

Publisher: Springer Nature

Persistent URL: https://hdl.handle.net/1721.1/123679

Version: Author's final manuscript: final author's manuscript post peer review, without publisher's formatting or copy editing

Terms of Use: Article is made available in accordance with the publisher's policy and may be subject to US copyright law. Please refer to the publisher's site for terms of use. 


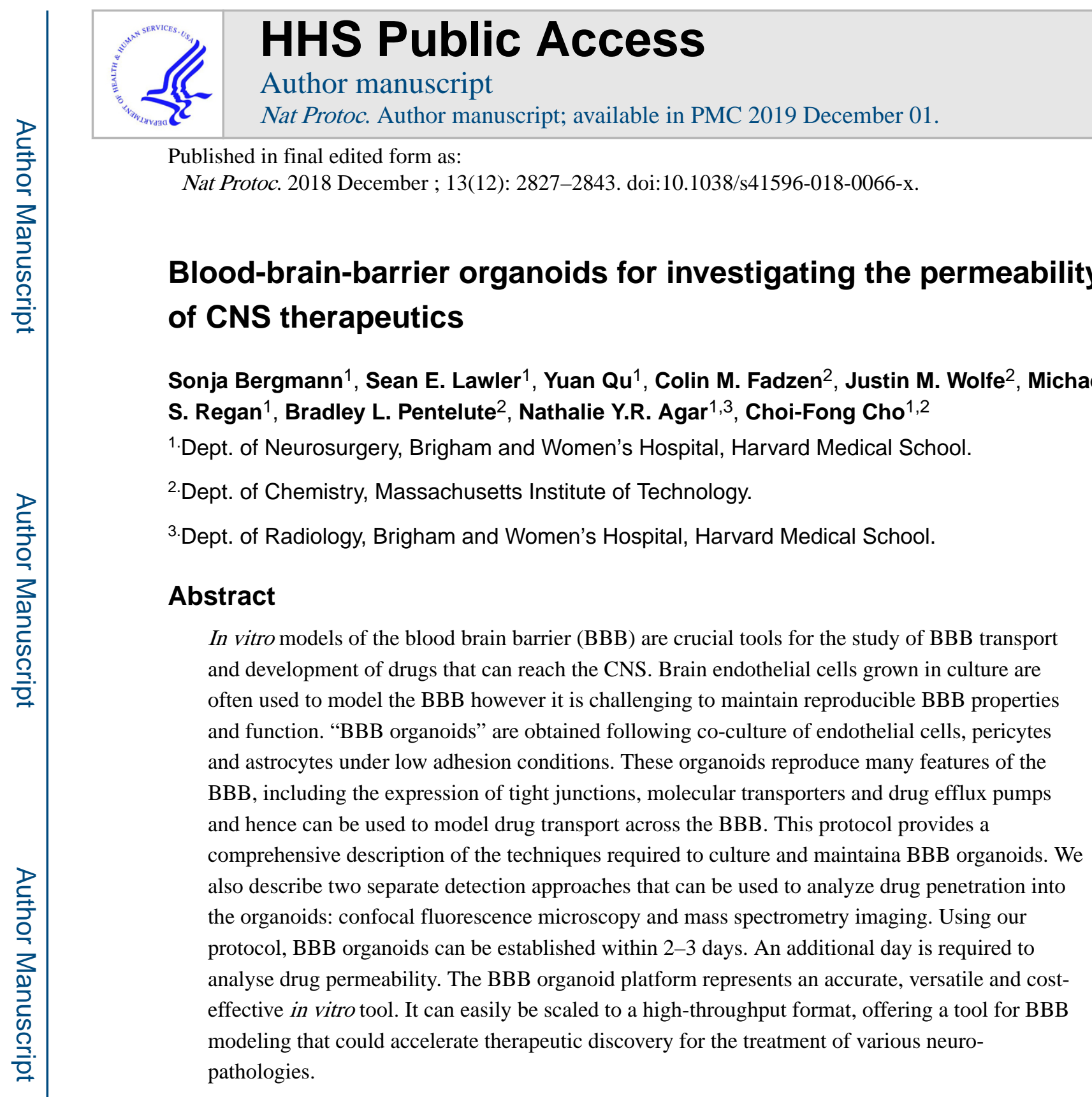

\section{EDITORIAL SUMMARY}

Endothelial cells, pericytes and astrocytes are co-cultured to generate organoids that reproduce many features of the blood-brain-barrier. This protocol also describes how to analyse drug penetration into the organoids.

\section{Keywords}

blood-brain barrier (BBB); drug delivery; permeability; in vitro model; organoid

Correspondence: Choi-Fong Cho, ccho@bwh.harvard.edu.

AUTHOR CONTRIBUTION STATEMENT C.-F.C, N.Y.R.A and S.E.L. designed the research. C.-F.C performed the experiments. S.B. and C.-F.C. analyzed the data. S.B., S.E.L., M.S.R. and C.-F.C. wrote the manuscript. C.M.F, J.M.W. and C.-F.C. revised the manuscript. All authors read and approved the final manuscript.

COMPETING INTERESTS STATEMENTS The authors declare that they have no competing financial interests. 


\section{INTRODUCTION}

Diseases of the central nervous system (CNS) represent a serious health burden. ${ }^{1,2}$ Their treatment is severely hampered by the blood brain barrier (BBB), a highly specialized arrangement of the vasculature that nature has evolved to regulate the entry of molecules into the brain and to provide protection from potentially harmful pathogens that enter the bloodstream. Unfortunately, the BBB also restricts the entry of the great majority of known therapeutics from the blood into the CNS. ${ }^{3}$ However, the BBB does permit the passage of nutrients and other molecules necessary for CNS function; thus is permeable to some molecules. The ability to model, study and understand the BBB could lead to the identification of approaches to enable transport of pharmacological agents into the brain, which could significantly advance therapy for many neuropathologies.

The BBB is comprised mainly of brain endothelial cells held closely together by tight junctions, and additional cell types with which the endothelial cells interact (including pericytes, microglial, neuron and astrocytic end-feet). ${ }^{4,5}$ When co-cultured under lowadherence conditions, human primary brain endothelial cells, astrocytes and pericytes assemble three-dimensional spheroidal structures comprised of astrocytes in the core with brain endothelial cells and pericytes encasing the exterior surface ${ }^{6,7}$. We have previously demonstrated that these multicellular BBB organoids can be used as a reliable and predictive in vitro platform to analyse and screen for brain-penetrating compounds. ${ }^{6}$ In this protocol we describe how to establish $\mathrm{BBB}$ organoids and use them to assess whether compounds can cross the BBB.

\section{The blood-brain-barrier in vivo}

Molecular entry through the BBB is strictly regulated in several ways.

- The tight junctions between endothelial cells prevent transcellular passage of molecules between the bloodstream and CNS tissues ${ }^{8}$.

- $\quad$ Specific molecular transporters shuttle essential molecules, such as glucose and amino acids across the $\mathrm{BBB}^{9,10}$.

- Transcytosis, in which a specific receptor binds to its ligand in the circulation on the luminal (blood-facing) side of the barrier and carries it to the abluminal (brain-facing) side enables transfer of other specific ligands. Transferrin and lowdensity lipoprotein (LDL) are examples of ligands that bind their respective receptors and enter the brain via this mechanism ${ }^{11}$.

- $\quad$ Drug efflux pumps, such as P-glycoprotein $(\mathrm{PgP})$ provide an additional layer of regulation by actively excluding molecules from the brain. ${ }^{12}$

Several approaches can be used to increase the permeability of the BBB. Physical approaches, such as osmotic shock using mannitol and focused ultrasound (FUS) nonselectively disrupt tight junctions transiently to allow unrestricted entry of vasculature components into the CNS. ${ }^{13,14}$ Other more selective strategies have been employed, such as the "Trojan horse" approach which exploits specific ligands as transport vehicles to hijack transcytosis receptors of the BBB. These include a 19 amino acid peptide called angiopep, 
which binds to the LRP1 receptor. ${ }^{15}$ Other promising approaches include the use of cellpenetrating peptides (CPPs) with the ability to readily cross membranes. ${ }^{16}$ Such approaches could be used to tag drugs, antibodies and nanoparticles for CNS delivery. However, none of these strategies is broadly used clinically at present, and drug delivery across the BBB remains a formidable challenge. Whilst neurosurgical procedures can be employed for direct intracranial injection of drugs into the brain, these methods are invasive and associated with greater risk to the patient. The passage of drugs across the BBB is dependent on various factors including molecular weight, hydrophobicity and polarity. Algorithms have been developed to help predict passage across the $\mathrm{BBB}$, but these are not reliable and drugs intended for clinical application need to be tested empirically. ${ }^{17}$

\section{Modelling the BBB}

In vitro models that can accurately mimic the $\mathrm{BBB}$ in culture are crucial tools for the development of brain-permeable drugs at the pre-clinical level. Such models would facilitate the development of new and optimized approaches to transport drugs across the BBB, as well as improve the ability to predict which therapeutic compounds would cross the BBB. In vitro BBB modeling has been developed since the $1980 \mathrm{~s},{ }^{18}$ although reproducing key BBB properties ex vivo remains challenging. Many researchers have used the transwell system to model the BBB. In the most commonly used transwell system, brain endothelial cells are cultured within the apical (top) side of the transwell insert, and astrocytes and pericytes are co-cultured on the basal (bottom) side of the setup. ${ }^{19,20}$ More recently, the incorporation of endothelial cells from cord blood-derived hematopoietic stem cells or human induced pluripotent stem cells (hiPSCs) has also been explored. ${ }^{21,22}$ While these platforms have proven useful for affinity binding assays and the study of signalling, transmigratory processes and BBB-immune cell interactions, ${ }^{23}$ these models are oversimplified and are associated with many well-known challenges. ${ }^{24}$ For example, it has been documented that the endothelial monolayer cultured on the transwell insert tends to exhibit various degrees of irregularity (multiple layers of endothelial cells, or small holes in the monolayer in certain areas) that might confer leakiness and significantly affect the experimental results. ${ }^{24}$ Microfluidic devices to simulate a more dynamic and realistic representation of the BBB by introducing blood flow to model the shear stress that occurs at the BBB have been developed to further improve physiological characteristics of the BBB in culture, ${ }^{25,26}$. A disadvantage of these systems is that specialized equipment and skillsets are required to build the platforms, hindering their broad application in the neuroscience community.

We recently introduced multicellular (human primary brain endothelial cells, astrocytes and pericytes) $\mathrm{BBB}$ organoids as a reliable and predictive in vitro analysis and screening platform for brain-penetrating compounds. ${ }^{6}$ A key feature of our system is that all the cell types are in direct contact with one another (unlike in other previously established models), which has been demonstrated to be crucial for the maintenance of BBB integrity. ${ }^{27}$ We have shown that each organoid displays enhanced BBB characteristics (including increased tight junctions, adherens junctions, and efflux pump expression) compared with endothelial cells cultured in the transwell system. ${ }^{6}$ Importantly, the presence of additional barrier functions within the organoids, such as lack of paracellular permeability, high drug efflux activity and receptor-mediated transcytosis (via the LRP-1 receptor) have been demonstrated. ${ }^{6}$. To our 
knowledge the BBB organoids currently offer the highest-throughput BBB modelling platform available owing to their miniature size and ease of culture. Hundreds of organoids can be used to simultaneously investigate numerous therapeutic compounds, and the throughput can be further enhanced through automated robotics-assisted detection techniques. We used these organoids to develop various new BBB-penetrating peptides, and confirmed their permeability in vivo. ${ }^{6,28}$ Organoid growth does not require external scaffold material. Although our approach does not incorporate flow (such as that incorporated in a microfluidic chamber), shear stress is introduced into the platform through constant rotation of the organoids at a regulated speed whilst the organoids are incubated with the test compound(s).

This protocol provides a detailed description of how to culture, maintain and employ the $\mathrm{BBB}$ organoids for investigating the BBB permeability of a given compound. However, whilst this platform is useful for rapid and predictive screening of drug permeability across the BBB, findings obtained using this in vitro tool should also be complemented with in vivo studies.

Experimental design: Brain endothelial cells, pericytes and astrocytes are cultured together in low-adherence condition in a 1:1:1 ratio, and allowed to self-assemble into multicellular spheroids over a $24-48$ hour period. The quality of established organoids should be assessed under a microscope before use. Organoids that have formed properly can be pooled together in working culture medium, and then incubated with the test compound of interest. We have incubated organoids for differing lengths of time (1-24h). The concentration and period of incubation need to be optimized for a given drug (it is also advisable to include proper positive and negative controls in all experiments). After incubation, the organoids are washed thoroughly, and processed to enable evaluation of compound permeability. We describe how to assess permeability using two different detection strategies: Confocal microscopy for fluorescently labelled compounds, as well as matrix-assisted laser desorption/ionization (MALDI) mass spectrometry imaging for nonfluorescent small molecules. Typically, the utility of one type of detection method is sufficient for a given compound, though both methods can be used to provide additional verification of a result if necessary. Confocal microscopy tends to be the preferred methodology, as it offers advantages such as better detection sensitive, higher throughput and fewer tissue preparation steps compared to mass spectrometry. Furthermore, the fluorescence detection route allows researchers to co-incubate their compound of interest with fluorescent-dextran as an internal negative control to assess the integrity of the BBB organoids. However, as most drugs are not inherently fluorescent, the use of mass spectrometry imaging for detection might be the only option. In the anticipated results section we show the results obtained following the analysis of angiopep-2 and BKM-120

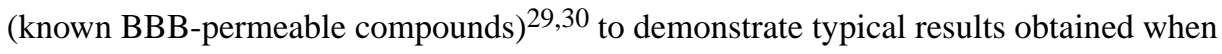
using this protocol. When considering the study of toxic drugs using this platform, the preservation of organoid integrity and function should be investigated by analysing the ability of the organoids to exclude dextran in the presence of the drug. 


\section{MATERIALS}

\section{REAGENTS}

- 2,5-dihydroxybenzoic acid (Sigma-Aldrich, cat. no. 149357)! CAUTION Harmful if swallowed. Causes skin, eye and respiratory irritation. Keep away from heat, sparks and open flames.

- $\quad$ Absolute ethanol (Fisher Scientific, cat. no. BP28184)! CAUTION Harmful if swallowed. Causes skin, eye and respiratory irritation. Keep away from heat, sparks and open flames.

- $\quad$ Agarose LE (Goldbio, cat. no. A-201-1000)

- Ammonium acetate (Sigma Aldrich, cat. no. A1542)

- $\quad$ Astrocyte growth medium AGM-2 BulletKit (includes $500 \mathrm{~mL}$ of astrocyte basal medium (ABM), recombinant human epidermal growth factor (rhEGF), insulin, ascorbic acid, GA-100, L-glutamine, fetal bovine serum (FBS)) (Lonza, cat. no. CC-4123)

- Dulbecco's phosphate-buffered saline (D-PBS) (GIBCO, cat. no. 14190-144)

- $\quad$ Endothelial cell medium complete kit (ECM) (includes $500 \mathrm{~mL}$ endothelial basal medium (EBM), $25 \mathrm{~mL}$ FBS, $5 \mathrm{~mL}$ of endothelial cell growth supplement (ECGS), $5 \mathrm{~mL}$ of penicillin/streptomycin solution) (ScienCell, cat. no. 1001).

- Endothelial growth medium EGM-2 BulletKit (includes $500 \mathrm{~mL}$ of endothelial basal medium, human epidermal growth factors (hEGF), hydrocortisone, GA-100, FBS, vascular endothelial growth factor (VEGF), human fibroblast growth factor B (hFGF-B), insulin like growth factor -1 ( $\mathrm{R}^{3}$-IGF-1), ascorbic acid and heparin) (Lonza, cat. no. CC-3162).

- $\quad$ Eosin Y solution (Sigma Aldrich, cat. no. HT110216)! CAUTION Causes eye irritation. Keep from heat, sparks and open flames.

- $\quad$ Fluorescein isothiocyanate (FITC)-dextran (70 kDa) (Sigma-Aldrich, cat. no. 46945) ! CAUTION Causes skin, eye and respiratory irritation. Use only in wellventilated areas.

- $\quad$ Formaldehyde solution 37\% (v/v) (Sigma Aldrich, cat. no. 252549) ! CAUTION Harmful if swallowed or inhaled. Causes skin, eye and respiratory irritation. Keep away from heat, open flames and oxidizers.

- $\quad$ Hoechst 33342 (Life technology, cat. no. H3570).

- $\quad$ Human AB serum (Valley Biomedical, cat. no. HP1022).

- $\quad$ Human brain microvascular pericytes (HBVP) (ScienCell, cat. no. 1200)! CAUTION Cell lines should be checked regularly to ensure they are authentic and free of mycoplasma. We check authenticity regularly by commercial STR at Yale University (Laboratory of Virology and Epidemiology) or IDEXX BioResearch. 
- $\quad$ Primary human astrocytes (Lonza, cat. No. CC-2565)! CAUTION Cell lines should be checked regularly to ensure they are authentic and free of mycoplasma. We check authenticity regularly by commercial STR.

- Human cerebral microvascular endothelial cell (hCMEC/D3) (Cedarlane, cat. no. CLU512) or primary human brain microvascular endothelial cells (HBMEC) (ScienCell, cat. no. 1000)! CAUTION Cell lines should be checked regularly to ensure they are authentic and free of mycoplasma. We check authenticity regularly by commercial STR.

- $\quad$ ! CRITICAL: Organoids can be established using either the immortalized hCMEC/D3 or the primary HBMEC cells. ${ }^{6}$ The 'anticipated results' section shows the utility of organoids made with hCMEC/D3 cells.

- Mayer's hematoxylin solution (Sigma Aldrich, cat. no. MHS16) ! CAUTION Harmful if swallowed, Causes skin, eye and respiratory irritation. Keep away from heat, sparks and open flames.

- $\quad$ Methanol high-performance liquid chromatography (HPLC) grade (Fisher Scientific, cat. no. A452-4)

- Mounting medium (Thermo Fisher Scientific, cat. no. 4112APG) ! CAUTION Harmful if swallowed, inhaled or absorbed through skin. Keep away from heat, sparks and open flames.

- $\quad$ Pericyte medium (includes $500 \mathrm{~mL}$ of pericyte basal medium, $10 \mathrm{~mL}$ of FBS, 5 $\mathrm{mL}$ of pericyte growth supplements (PGS) and $5 \mathrm{~mL}$ of penicillin/streptomycin solution) (ScienCell, cat. no. 1201).

- $\quad$ Anti-CD31 rabbit antibody (Abcam, cat. no. ab28364).

- $\quad$ Anti-VE-Cadherin monoclonal antibody (Cell Signaling Technology, cat. no. 2500P).

- $\quad$ Anti-NG2 rabbit antibody (Millipore, cat. no. ab5320).

- $\quad$ Anti-GFAP mouse antibody (Sigma-Aldrich, cat. no. G3893).

- $\quad$ Anti-ZO-1 mouse monoclonal antibody (Life Technologies, cat. no. 33-9100).

- $\quad$ Anti-P Glycoprotein antibody [4E3] (Abcam, cat. no. ab10333).

- Tetramethylrhodamine isothiocyanate (TRITC)- dextran (4,400 Da) (SigmaAldrich, cat. no. T1037)! CAUTION Harmful if swallowed or inhaled. Causes skin, eye and respiratory irritation.

- $\quad$ Tissue-Tek optimum cutting temperature (O.C.T.) compound (Fisher Scientific, cat. no. NC9636948)

- $\quad$ Trifluoroacetic acid (TFA) (Sigma Aldrich, cat. no. T6508) ! CAUTION Harmful if swallowed, inhaled or absorbed through skin. Wash skin thoroughly after handling and use only in well-ventilated areas. 
- $\quad$ Triton X-100 (Sigma Aldrich, cat. no. T8532)! CAUTION Harmful if swallowed. Causes skin and eye irritation.

- $\quad$ Trypsin-Ethylenediaminetetraacetic acid (EDTA) $0.05 \%$ (GIBCO, cat. no. 25300-054).

- Water HPLC (Fisher, cat. No. W54).

- Xylene (Sigma Aldrich, cat. no. 534056) ! CAUTION Harmful if swallowed, inhaled or absorbed through skin. Cause skin and respiratory irritation. Keep away from heat, sparks and open flames.

- $\quad$ BKM-120 (Selleckchem, cat. no. S2247). CAUTION Could be harmful if swallowed, inhaled or absorbed through skin.

- $\quad$ Dabrafenib (Selleckchem, cat. no. S2807).

- $\quad$ Peptides of interest. The example results shown in this manuscript were following exposure of BBB organoids to Angiopep-2

(TFFYGGSRGKRNNFKTEEY) and a corresponding scramble control peptide (GNYTSRFEREYGKFNKFGT). These peptides were synthesized and purified in the Pentelute laboratory (Dept. of Chemistry, Massachusetts Institute of Technology). These peptides were labeled with tetramethylrhodamine (TAMRA) as described in Box 2.

\section{EQUIPMENT}

- $\quad 0.6 \mathrm{~mL}$ microcentrifuge tubes (Thermofisher Scientific, cat. no. 05-408-120)

- $\quad 1.5 \mathrm{~mL}$ microcentrifuge tube (Eppendorf, cat. no. 022431081)

- $\quad 37^{\circ} \mathrm{C}$ incubator (Fisher Scientific, cat. no. 11-690-650D)

- $\quad$ 8-channel pipette (Bioexpress, cat. no. P-4920-10)

- $\quad$ 9.4 T SaloriX XR Fourier transform ion cyclotron resonance (Bruker Daltonics; software: FlexImaging 4.1)

- $\quad$ 96-well plates (Greiner Bio-one, cat. no. 655180)

- $\quad$ Axio Observer Z1 confocal microscope (Zeiss; software: Zen 2012 black edition)

- $\quad$ Cell + yellow-vented cap tissue culture flask T75 or T175 (Sarstedt, cat. no. 833911302/833912302)

- $\quad$ Cover glasses (Fisher Scientific, cat. no. 12-548-5P)

- $\quad$ Cryostat (Thermo Fisher Scientific, Model MICROM HM550)

- $\quad$ Digital multimeter (Fluke, Model 179)

- $\quad$ Disposable glass pasteur pipettes (VWR, cat. no. 14673-010)

- Disposable serological pipettes (5 mL, $10 \mathrm{~mL}, 25 \mathrm{~mL}$ ) (Greiner Bio-one, cat. no. 606180/607160/760160) 
- $\quad$ Filtered pipette tips $(10 \mu \mathrm{L}, 200 \mu \mathrm{L}, 1250 \mu \mathrm{L})(\mathrm{VWR}$, cat. no. 10017032/-078/-082)

- $\quad$ Hemocytometer (Sigma-Aldrich, cat. no. Z359629-1EA)

- $\quad$ High purity nitrogen cylinder (Air Gas, cat. no. NI UHP300)

- Humidified $\mathrm{CO}_{2}$ incubator (Thermo Fisher Scientific, cat. no. 310)

- $\quad$ Indium tin oxide (ITO) slides (Bruker Daltonics, cat. no. 237001)

- Inverted light microscope (Nikon Eclipse TS100)

- $\quad$ Isotemp $37^{\circ} \mathrm{C}$ water bath (Fisher Scientific, Model 2223)

- $\quad$ Microtome blades (Sturkey, cat. no. D554X50)

- $\quad$ Microwave (Panasonic)

- $\quad$ Mini centrifuge (Midwest Scientific, cat. no. C1301)

- $\quad$ Nunc Lab-Tek II 8-well chambered coverglass (Thermofisher Scientific, cat. no. 155409)

- $\quad$ PCR tubes (Sigma Aldrich, cat. no. CLS6571-960EA)

- $\quad$ Pipet basin $50 \mathrm{~mL}$ (Fisherbrand, cat. no. 13-681-502)

- $\quad$ SterilGARD biosafety cabinet (tissue culture hood) (The Baker Company, Model SG403A-HE)

- $\quad$ Superfrost plus microscope slides (Fisher Scientific, cat. no. 12-550-15)

- TM Sprayer (HTX Technologies LLC)

- $\quad$ Tube rotator (Denville Scientific Inc., cat. no. H5700)

- Vacuum desiccator (Cole-parmer, cat. no. 42025)

\section{REAGENT SETUP}

- $1 \%(\mathrm{w} / \mathrm{v})$ agarose. Dissolve $0.5 \mathrm{~g}$ of agarose in $50 \mathrm{~mL}$ of MilliQ water or PBS. Heat up until completely dissolved. Prepare fresh agarose for every new experiment.

- Wash buffer for mass spectrometry imaging. Dissolve $0.12 \mathrm{~g}$ of ammonium acetate in $200 \mathrm{~mL}$ HPLC grade water, $\mathrm{pH}$ 7. Store bottle at $25^{\circ} \mathrm{C}$ or in the fridge for no longer than 6 months.

- 2,5-dihydroxybenzoic acid matrix (MALDI matrix). Dissolve 800mg of 2,5DHB $(160 \mathrm{mg} / \mathrm{mL})$.

\section{PROCEDURE}

In $5 \mathrm{~mL}$ of $70 / 30 \mathrm{Methanol} / \mathrm{H}_{2} \mathrm{O}$ and $0.1 \%$ TFA. Prepare fresh solution for every new experiment. 
- $\quad$ Endothelial growth medium (EGM-2 from Lonza) (for immortalized hCMEC/D3). Add all supplements and growth factors provided by the manufacturer (hEGF, hydrocortisone, GA-100, FBS, VEGF, hFGF-B, R ${ }^{3}$-IGF-1, ascorbic acid, heparin) to $500 \mathrm{~mL}$ of endothelial basal medium in a sterile tissue culture hood. Invert bottle a few times to ensure thorough mixing of all components. Store bottle at $2-8^{\circ} \mathrm{C}$ in the fridge for no longer than 6 weeks.

- Endothelial cell medium (ECM from ScienCell) (for primary HBMEC). Add $25 \mathrm{~mL}$ of FBS and $5 \mathrm{~mL}$ of endothelial cell growth supplement to $500 \mathrm{~mL}$ of endothelial basal medium. Invert bottle a few times to ensure thorough mixing of all components. Store bottle at $2-8^{\circ} \mathrm{C}$ in the fridge for no longer than 6 weeks.

- $\quad$ Astrocyte growth medium (AGM-2). Add all supplements and growth factors provided by the manufacturer (rhEGF, insulin, ascorbic acid, GA-100, Lglutamine, FBS) to $500 \mathrm{~mL}$ of astrocyte growth medium in a sterile tissue culture hood. Invert bottle a few times to ensure thorough mixing of all components. Store bottle at $2-8^{\circ} \mathrm{C}$ in the fridge for no longer than 6 weeks.

- $\quad$ Pericyte medium. Add $10 \mathrm{~mL}$ of FBS and $5 \mathrm{~mL}$ of pericyte growth supplement to $500 \mathrm{~mL}$ of pericyte basal medium in a sterile tissue culture hood. Invert bottle a few times to ensure thorough mixing of all components. Store bottle at $2-8^{\circ} \mathrm{C}$ in the fridge for no longer than 6 weeks.

- $\quad$ BBB working medium. For organoid formation using hCMEC/D3, add endothelial growth supplements hEGF, hydrocortisone, GA-100, hFGF-B, R ${ }^{3}$ IGF-1, ascorbic acid and heparin to $500 \mathrm{~mL}$ of endothelial basal medium (EBM from Lonza) in a sterile tissue culture hood. Supplement this medium with $2 \%$ human serum. Invert bottle a few times to ensure thorough mixing of all components. Store bottle at $2-8^{\circ} \mathrm{C}$ in the fridge for no longer than 6 weeks.! Critical Do not add FBS (since medium has already been supplemented with human serum) and VEGF (as VEGF will disrupt the tight junctions on the organoid surface) to this medium. ! Critical For organoid formation using primary HBMEC, add $2 \%$ human serum to endothelial basal medium (from ScienCell) and exclude supplementation with FBS and ECGS.

\section{Cell maintenance and initial preparation steps - TIMING 30-40 minutes}

CRITICAL Perform all tissue culture procedures within a ventilated class II biological safety cabinet or tissue culture hood.

1. Perform all tissue culture procedures within a ventilated class II biological safety cabinet or tissue culture hood. Maintain either immortalized human cerebral microvascular endothelial cells (hCMEC/D3) in endothelial growth medium (EGM-2) or primary human brain microvascular endothelial cells (HBMEC) in endothelial cell medium (ECM). Maintain primary human brain vascular pericytes (HBVP) in pericyte medium and primary human astrocytes in astrocyte growth medium (AGM-2). Culture each of these cell types in approximately 20 $\mathrm{mL}$ of cell culture medium in a T75 cell culture flask or in approximately $30 \mathrm{~mL}$ of cell culture medium in a T175 cell culture flask (Cell+ growth surface for 
sensitive adherent cells) with a yellow-vented cap in a humidified incubator at $37^{\circ} \mathrm{C}$ with $5 \% \mathrm{CO}_{2}$ and $95 \%$ natural air. Culture cells for a few days until they reach a confluency of approximately $80 \%$ (see bright field images in Fig. 1) in the appropriate cell culture medium. $\Delta$ CRITICAL STEP For experimental use, maintain astrocytes between passages 2 and 5, HBVP between passages 2 and 10, and HBMEC between passages 2 and 5. If required, passage cells when they reach $80 \%$ confluency by trypsinization (as described in steps 8-11) and split at a 1:10 ratio for each cell type. Upon obtaining a new stock, or as the cell passage reaches its higher limit, confirm the identity of each cell type by immunofluorescence staining of their cell-specific marker(s) (a marker for astrocytes is GFAP; marker for HBVP is NG2; and markers for endothelial cells are CD31 and VE-cadherin) (Fig. 1). Cell identity and authentication can also be confirmed by transmission electron microscopy. ${ }^{6}$

\section{? TROUBLESHOOTING}

2. Pre-warm the appropriate BBB working medium, D-PBS, EGM-2 (or ECM), AGM-2, pericyte medium and $0.05 \%$ Trypsin-EDTA in a clean water bath at $37^{\circ} \mathrm{C}$ for $\sim 30$ minutes.

\section{Preparation of low-adhesion agarose plate - TIMING 25-35 minutes}

3. Prepare $1 \%(\mathrm{w} / \mathrm{v})$ agarose by mixing $0.5 \mathrm{~g}$ of molecular biology grade agarose in $50 \mathrm{~mL}$ of MilliQ water or D-PBS in a conical flask.

4. Microwave the mixture for 1-2 minutes (with pauses in between to prevent boiling over) until the agarose is completely dissolved.

5. Transfer the flask containing the melted agarose into a sterile tissue culture hood and pour the solution into a $50-\mathrm{mL}$ pipette basin.

6. Pipette $50 \mu \mathrm{L}$ of agarose into each well of a 96-well plate while it is still hot using sterile pipette tips and a multi-channel pipette.

CRITICAL STEP Work quickly at this step to avoid cooling/solidification of the agarose solution during pipetting, as this can lead to uneven agarose coating within each well.

7. Set the plate aside to cool at room temperature $\left(20-24^{\circ} \mathrm{C}\right)$ and allow the agarose to solidify for $\sim 15$ minutes. If the plate is not used immediately, add $\sim 50 \mu \mathrm{m}$ of working media into each well to prevent the agarose from drying, and store the plate at $4^{\circ} \mathrm{C}$ for up to $3 \mathrm{hrs}$.

\section{? TROUBLESHOOTING}

\section{Trypsinization of cells - TIMING 20-30 minutes}

8. Transfer the flasks containing hCMEC/D3 (or HBMEC), HBVP and astrocytes from the incubator into the tissue culture hood. Aspirate the media from each cell culture flask. 
9. Wash the cells gently with $10 \mathrm{~mL}$ of pre-warmed D-PBS, and then aspirate the solution.

10. Add $1-1.5 \mathrm{~mL}$ of $0.05 \%$ Trypsin-EDTA to each flask, and gently rock each flask back and forth to ensure that the trypsin solution is properly distributed over all the cells on the surface of the flask. Place the flasks back in the $37^{\circ} \mathrm{C}$ incubator for 2-5 minutes or until cells begin to detach from the surface of the flask. If necessary tap dish to help release the cells.

11. Add $9 \mathrm{~mL}$ of BBB working medium to each flask to resuspend the cells, generating a working stock for each cell type.

12. Count each cell type using a hemocytometer and determine the concentration of each working stock.

\section{Organoid culture - TIMING 48 hrs}

13. Calculate the volume needed to seed $1.5 \times 10^{3}$ cells of hCMEC/D3 (or HBMEC), HBVP and astrocytes. To achieve a volume of $200 \mu \mathrm{L}$ per well in a 96-well agarose plate, subtract the total cell volume from $200 \mu \mathrm{L}$, and pipette that amount of BBB working medium into each well using a multi-channel pipette. Then, add the appropriate volume of each cell type using a multi-channel pipette into each well containing the BBB working medium. The final volume in each well should be $200 \mu \mathrm{L}$.

$\triangle$ CRITICAL STEP To ensure proper cell seeding, assess each well under an inverted light microscope after each seeding step. To reduce the 'edge effect' in a 96-well plate, where the level of evaporation at the edge wells is much higher, fill the outer edge wells with $200 \mu \mathrm{L}$ of either D-PBS or BBB working medium to act as a moisture barrier, and use only the inner wells of the plate for culturing the BBB organoids.

\section{? TROUBLESHOOTING}

14. Incubate the plate at $37^{\circ} \mathrm{C}$ in a humidified $\mathrm{CO}_{2}$ incubator for $48 \mathrm{~h}$. The organoids should assemble properly into compact spheroids within 12-24 hrs (Fig. 2a). After the $48 \mathrm{hrs}$, assess the organoid quality in each well prior to collecting them for experimental purposes (see Fig. $2 \mathrm{~b}$ as a general guideline). Proceed to the next steps only with organoids that have assembled properly. Collect each good organoid by pipetting, and pool them together in a microcentrifuge tube for experimental use (Fig. 2c). CRITICAL STEP Organoid quality can be further assessed by immuno-staining example organoids for expression of markers of the $\mathrm{BBB}$, such as tight junctions (ZO-1) and efflux pump (P-glycoprotein) as illustrated in Fig. 2d and e.

\section{Analysis of BBB-penetrating compounds - TIMING between 1 to $24 \mathrm{hrs}$}

15. Either pool multiple organoids together in microcentrifuge tubes in BBB working medium for subsequent confocal fluorescence microscopy (option A) or matrix-assisted laser desorption ionization (MALDI) mass spectrometry imaging 
(MSI; option B) or treat a single organoid separately with a test compound for confocal fluorescence microscopy (option C). When choosing which method to use bear in mind that confocal fluorescence microscopy can only be done if the compound of interest is inherently fluorescent or is labeled with a fluorophore. For non-fluorescent molecules, MALDI mass spectrometry imaging is an appropriate alternative. The use of multiple organoids pooled together (shown in Fig. 2b) allows them to be used simultaneously to test a given compound. The use of pooled organoids is best used for short-span experiments, when organoids are incubated with the test compound for only a few hours. For experiments where long-term incubation (over days) is needed, a single organoid can be treated separately with a test compound whilst in culture in a 96-well plate (option C). Although this method is slightly more laborious and utilizes a higher amount of test reagent, this is necessary to prevent the organoids from merging/ clumping together in a pooled environment over time. Additionally, the test compound can be co-incubated with fluorescent high-molecular weight dextran as an internal control to ensure that the compound of interest does not disrupt the surface barrier of the organoid. If a high level of the test compound is detected within a functional organoid that can exclude dextran, this would suggest true BBB permeability.

\section{A) Treatment of pooled organoids for confocal fluorescence microscopy}

i. Pool 7-10 organoids per group into a $0.6-\mathrm{mL}$ microcentrifuge tube in BBB working medium by transferring single organoids from the agarose-coated 96well plate using a $200-\mu \mathrm{L}$ pipette tip.

ii. Gently spin down organoids using a table-top mini-centrifuge for 2-3 seconds (at approximately 100-300 xg), and aspirate medium carefully without disturbing the organoids. Add $500 \mu \mathrm{L}$ of fresh BBB working medium into the tube.

iii. Pipette the test compound (fluorescently-labeled) into each tube containing the organoids to a desired final concentration (typical range is $1-20 \mu \mathrm{M}$ ) and mix well by inverting the tube. If desired, add also a fluorescently-labeled highmolecular weight dextran to a final concentration of $10 \mu \mathrm{g} / \mathrm{mL}$ as an internal control.

\section{? TROUBLESHOOTING}

iv. Incubate organoids for the desired duration, (typically between 1-24 hrs) under constant rotation in a $37^{\circ} \mathrm{C}$ incubator.

$\triangle$ CRITICAL STEP It is necessary to test a diverse range of working concentration and duration of incubation period for each test compound to determine the optimal parameters for analyzing BBB permeability without disrupting the structure/function of the organoids. This can be examined by coincubation of the test compound with dextran (as described above). This step is particularly important if the test compound is known to be toxic to cells (e.g. anti-cancer drugs). Table 1 below summarizes a list of peptides that have been previously tested for BBB permeability using the organoid model. 


\section{? TROUBLESHOOTING}

v. Gently spin organoids down using a mini-centrifuge for 2-3 seconds (approximately $100-300 \mathrm{xg}$ ) and carefully aspirate the solution. Add $500 \mu \mathrm{L}$ of BBB working medium or D-PBS to wash the organoids/remove any excess test compound. Repeat washing step at least $3 \mathrm{x}$ by centrifuging and washing organoids in working medium or D-PBS each time.

\section{B) Treatment of pooled organoids for MALDI MSI}

i. Collect/pool 150-200 organoids per group into a 1.5-mL microcentrifuge tube in BBB working medium using a micropipette. CRITICAL STEP Organoid cryosections are analyzed to examine whether a test compound has penetrated the surface barrier and entered the core. This requires a sizable pellet of pooled organoids for each test group to ease the cryo-sectioning process (see below).

ii. Gently spin organoids down 2-3 seconds (at approximately 100-300 xg) and aspirate the medium carefully. Add $1 \mathrm{~mL}$ of fresh BBB working medium into the tube.

iii. Add the test compound (of known molecular weight) into the tube to the desired final concentration (typical range is $1-10 \mu \mathrm{M}$ ) and mix well by inverting the tube.

$\triangle$ CRITICAL STEP As described for option A, determine if the desired concentration of the test compound would disrupt the integrity of the BBB surface barrier by co-incubation with a high-molecular weight dextran.

\section{? TROUBLESHOOTING}

iv. Incubate the organoids at $37^{\circ} \mathrm{C}$ in an incubator under constant rotation. The incubation period is typically between 1-24 hrs, though this parameter should be optimized for each compound.

\section{? TROUBLESHOOTING}

v. Gently spin organoids down using a mini-centrifuge for 2-3 seconds (approximately 100-300 xg) and carefully aspirate the solution. Add $500 \mu \mathrm{L} 7.5$ $\mathrm{mM}$ ammonium acetate wash buffer to wash the organoids/remove any excess test compound. Repeat this washing step 5x.

\section{C) Treatment of individual organoids in a 96-well plate}

i. Pipette the test compound into each well containing a single organoid at a desired concentration. Pipette up/down gently to ensure proper mixing.

\section{? TROUBLESHOOTING}

ii. Incubate the plate in a humidified $\mathrm{CO}_{2}$ incubator at $37^{\circ} \mathrm{C}$ (typical duration is $1-3$ days). Change the working media every 2 days if a longer incubation period is required. 
iii. Collect organoids into a $0.6-\mathrm{mL}$ microcentrifuge tube using a micropipette. Organoids from the same test group can be pooled together in the same tube.

iv. Gently spin organoids down using a mini-centrifuge for 2-3 seconds (approximately 100-300 xg) and carefully aspirate the solution. Add $500 \mu \mathrm{L}$ of BBB working medium or D-PBS to wash the organoids to remove any excess test compound. Repeat washing step at least $3 x$ by centrifuging and washing the organoids in working medium or D-PBS each time.

16. Use fluorescence microscopy (option A) or MALDI MSI (option B) to analyze compound permeability across the BBB. Option A below can be carried out after step 15 option A or C. Option B below can be carried out after step 15 option B. BBB permeability within a single organoid can be quantitatively analyzed by confocal imaging.

\section{A) Imaging using confocal fluorescence microscopy}

i. Remove/aspirate BBB working medium or PBS from pooled organoids.

ii. Add $450 \mu \mathrm{L}$ of $3.7 \%(\mathrm{v} / \mathrm{v})$ formaldehyde to fix the organoids. Lay the microcentrifuge tube sideways, spreading the organoids distribution to ensure that they are not touching each other to prevent organoid merging/aggregation. Incubate for 15 minutes at room temperature.

iii. Gently spin organoids down using a mini-centrifuge for 2-3 seconds (approximately 100-300 xg) and carefully aspirate the solution. Add $500 \mu \mathrm{L}$ of BBB working medium or D-PBS to wash the organoids/remove any excess test compound. Repeat washing step at least $3 x$ by centrifuging and washing spheroids in working medium or D-PBS each time.

iv. If desired, add DAPI or Hoechst dye (1:1000 dilution) to stain the nuclei of the cells on the outer organoid surface. Incubate for 1 min and wash $3 \mathrm{x}$ with $500 \mu \mathrm{L}$ of BBB working medium or D-PBS by spinning them down for 2-3 seconds using a mini-centrifuge (approximately 100-300 xg).

v. Coat the inner wall of a $200-\mu \mathrm{L}$ pipette tip with D-PBS containing $0.1-0.2 \%$ (v/v) Triton-X by pipetting up/down a few times (for $\sim 5$ seconds), and then use the tip to transfer the washed organoids to a chambered coverglass for imaging.

$\triangle$ CRITICAL STEP During the transfer process, organoids can stick to the inner wall of the pipette tip, causing them to be irretrievable for further analysis. Coating the inner wall of the pipette tip with $0.1-0.2 \%(\mathrm{v} / \mathrm{v})$ Triton-X in D-PBS solution prior to transferring the organoids prevents this.

\section{? TROUBLESHOOTING}

vi. To analyze compound permeability using confocal fluorescence microscopy, capture z-stack images starting from the outer most surface of the organoid towards the core using a 20x objective (up to approximately $100 \mu \mathrm{m}$ deep, or until the fluorescence level has degraded). If a two- or multi-photon microscope is used, a greater imaging depth $(>100 \mu \mathrm{m})$ can be achieved. 
$\triangle$ CRITICAL STEP For quantitative analysis, it is important to adjust the microscope settings (laser power, exposure time and/or gain) appropriately so that the signal intensity of the test compound at the desired depth of the organoid would fit within the dynamic range of the detector. Settings that are too low (below detectable threshold) will not display any fluorescence, while settings that are too high will result in saturated pixels that are not quantifiable.

\section{B) Imaging using MALDI MSI}

i. Start the process of chilling the cryostat chamber and specimen holder to approximately $-26^{\circ} \mathrm{C}$ so these are at an appropriate temperature ready for step v.

ii. Coat pipette tip with D-PBS containing $0.1-0.2 \%(\mathrm{v} / \mathrm{v})$ Triton-X by pipetting up/ down a few times (for $\sim 5$ seconds), and transfer the organoids to a $0.2-\mathrm{mL}$ PCR tube in $200 \mu \mathrm{L}$ of ammonium acetate buffer.

$\triangle$ CRITICAL STEP During the transfer process, organoids can stick to the inner wall of the pipette tip, causing them to be irretrievable for further analysis. To prevent this, coat the inner wall of the pipette tip with $0.1-0.2 \%$ (v/v) Triton$\mathrm{X}$ in D-PBS solution prior to transferring the organoids.

\section{? TROUBLESHOOTING}

iii. Gently spin the organoids down for 2-3 seconds at approximately 100-300 xg to pool them at the bottom the PCR tube.

iv. Flash freeze the organoids by placing the tube in a dry ice/ethanol bath for a few minutes until the buffer solution is completely frozen.

v. Mount the top of the frozen tube onto a sample disc/holder using the Tissue-Tek O.C.T medium and place the setup on dry ice until the O.C.T medium is frozen (Fig. 5a). Then, clamp the sample disc containing the frozen tube of organoids onto the sample stage of the cryostat (Fig. 5a), with both the chamber and specimen holder chilled at approximately $-26^{\circ} \mathrm{C}$.

vi. Cryo-section the bottom of the frozen tube containing the organoids into $12 \mu \mathrm{m}$ slices, and thaw-mount the organoid tissue onto an indium tin oxide (ITO) coated microscopic slide for MALDI MSI. Also mount serial sections onto a regular positively charged glass slide for haematoxylin and eosin (H\&E) staining to verify the quality of the tissue sections.

$\triangle$ CRITICAL STEP To ensure that the sections are mounted onto the correct side of the ITO-coated slides, measure the resistance on both sides of the slide using a voltage-meter. ITO coating causes electrical resistance, while the uncoated side is not conductive (see Fig. 5b). Ensure that sections are mounted onto the ITO coated side.

vii. Stain tissue on the positively charged slides with $H \& E$ to confirm the presence of BBB organoid tissue (see Box 1). 
viii. Place ITO slides containing the tissue sections into a desiccator for at least 15 minutes.

ix. For MALDI MS imaging, dissolve the MALDI matrix (2,5-dihydroxybenzoic matrix) 70:30 (v/v) in methanol containing $0.1 \%(\mathrm{v} / \mathrm{v})$ TFA.

x. Apply $1.91 \mathrm{~mL}$ matrix solution across the tissue sections using a TM-sprayer under the following conditions:

Flow rate, $180 \mathrm{ml} \mathrm{min}{ }^{-1}$; spray nozzle velocity, $1,200 \mathrm{~mm} \mathrm{~min}^{-1}$; spray nozzle temperature, $75^{\circ} \mathrm{C}$; nitrogen gas pressure, $10 \mathrm{psi}$; track spacing, $2 \mathrm{~mm}$; and number of passes, 2 .

xi. Acquire mass spectra using a Fourier transform ion cyclotron resonance (FTICR) mass spectrometer. Set pixel step size for the surface raster to a maximum of 30 $\mu \mathrm{m}$. Analyze compound using a positive ion mode targeting the molecule of interest with continuous accumulation of selected ions (CASI) in the corresponding mass range.

\section{Data analysis - TIMING 1-2 hrs}

17. Analyze compound permeability using option A if you have carried out confocal fluorescence microscopy and option B if you have carried out MALDI MSI.

\section{A) Quantification of z-stack images from confocal fluorescence microscopy}

-CRITICAL Quantification of acquired z-stack images can be performed by measuring the mean fluorescence intensity of the compound inside the organoid at a depth between approximately $50-100 \mu \mathrm{m}$ using ImageJ (Fig. 3).

i. Using the Fiji software (ImageJ), select the optical sections between approximately $50 \mu \mathrm{m}$ and $100 \mu \mathrm{m}$ depth.

ii. Determine the core area of each organoid by measuring $50 \mu \mathrm{m}$ from the surface. Use the scale bar as a reference to assess the distance appropriately.

iii. Add the selected region to the Region of Interest (ROI) Manager by clicking Edit $>$ Selections > Add to manager or by pressing Ctrl+ ' $\mathrm{T}$ '.

iv. Measure the mean fluorescence intensity (MFI) within the selected region by using Analyze > Measure or press Ctrl + 'M'.

v. Calculate the sum of the mean fluorescence intensity of these optical sections.

\section{B) Detection of molecular compounds from MALDI-FTICR Mass}

\section{Spectrometry Imaging-CRITICAL Detection of specific compounds acquired from} MALDI-MS images can be performed by analysis of the normalized Total Ion Current (TIC) intensity for specific mass-to-charge $(\mathrm{m} / \mathrm{z})$ ratios.

i. Set experimental parameters in FlexControl software (Bruker Daltonics) to positive ion mode by Continuous Accumulation of Selected Ions (CASI mode) in a mass range between $\mathrm{m} / \mathrm{z} 405-525$ and a laser intensity set to $25 \%$. Each mass spectrum is the sum of 250 laser shots at a laser frequency of $1000 \mathrm{~Hz}$. 
ii. Open IsotopePattern software (Bruker Daltonics) and use your knowledge of the chemical formula to calculate the $\mathrm{m} / \mathrm{z}$ ratio for the exact mass of the specific molecular species being detected. This ensures that the correct mass of the compound of interest is being targeted within the CASI window spectra as mentioned above.

iii. Import acquired data from the FTICR-MS into FlexImaging 4.0. Once data file is uploaded to FlexImaging 4.0, normalize the comprehensive mass spectra to TIC. This diminishes background signal and MS run bias that could affect downstream analysis.

iv. Select specific Regions of Interest (ROI) by outlining areas in flexImaging 4.0. Individual spectra for selected ROI's can be analyzed in separate window.

v. Using the exact mass-to-charge $\mathrm{m} / \mathrm{z}$ ratio for each molecular species as calculated in step ii. through IsotopePattern, investigate specific areas in the mass spectra. Targeting already known $\mathrm{m} / \mathrm{z}$ values for compounds of interest can be done by a mass search or manually by scrolling through the $\mathrm{x}$-axis $(\mathrm{m} / \mathrm{z})$ values and finding peaks with corresponding mass-to-charge ratios.

TIMING

This is an estimate for the screening of one compound

Steps 1-2: 30-40 minutes

Steps 3-7: 25-35 minutes

Steps 8-12: 20-30 minutes

Steps 13-14: $48 \mathrm{hrs}$

Step 15-16: 1 to $24 \mathrm{hrs}$

Step 17: 1-2 hrs

? TROUBLESHOOTING

See Table 3.

\section{ANTICIPATED RESULTS \\ Investigation of active transport across the BBB organoid surface via confocal microscopy.}

BBB organoids established using hCMEC/D3, pericytes and astrocytes should express cellspecific markers. Expression of these markers can be determined by immunofluorescence staining (for example Fig. 1). The example results we show here demonstrate that angiopep-2, a peptide ligand of the low density lipoprotein receptor-related protein-1 (LRP-1) receptor, which is known to cross the BBB via receptor mediated transcytosis ${ }^{15,29}$ penetrates the BBB organoids. For detection by confocal microscopy, angiopep- 2 as well as 
a corresponding scramble control peptide were labeled with a tetramethylrhodamine dye (Box 2). After incubation with the organoids, z-stack confocal fluorescence images were acquired to capture the level of accumulation of each compound within the organoid core. Permeability is quantified by measuring the total mean fluorescence signal intensity of the optical sections at a depth between $56-96 \mu \mathrm{m}$. As expected, we observed a high influx of angiopep-2 into the organoids compared with the scramble control peptide, while rhodamine-dextran and unconjugated rhodamine dye were undetectable (Fig.4a-c). We also demonstrated the permeability of $\operatorname{Bip}(1)$, a cell-penetrating peptide that can penetrate the BBB organoids in a time- and concentration-dependent manner (Fig. 4d, e). ${ }^{6}$

\section{Analysis of small-molecule drug transport using MALDI MSI}

MALDI mass spectrometry imaging can be used to analyze whether non-fluorescent molecules penetrate the BBB organoids. In our example results, we show data following incubation with phosphatidylinositol 3-kinase inhibitor BKM120, a molecule known to cross the $\mathrm{BBB},{ }^{30,33}$ and dabrafenib, an inhibitor of the threonine-protein kinase B-Raf with limited BBB penetration. ${ }^{34}$ Established organoids were incubated with $10 \mu \mathrm{M}$ of either drug for $24 \mathrm{hrs}$, and then cryo-sectioned for MS imaging. The tissue sections of the organoids were scanned, and MS data was acquired at a spatial resolution of $30 \mu \mathrm{m}$ to allow evaluation of drug penetration inside the organoids. A high level of BKM120 is seen in the BBB organoids, as would be expected given that BKM120 can penetrate the BBB, while dabrafenib were not detected (Fig. 5c).

\section{CONCLUSION}

The BBB organoid platform models the BBB in vitro, recapitulating key BBB properties and functions. This high-throughput and cost-effective approach can offer a paradigm shift for in vitro BBB modeling and investigation of brain-permeable molecules for a broad range of therapeutic application for CNS diseases.

\section{ACKNOWLEDGMENTS}

We acknowledge Haley Bridger and Hannah Schulze from the Brigham and Women's Research Institute for providing some assistance with photography.

\section{References}

1. DiLuca M \& Olesen J The cost of brain diseases: a burden or a challenge? Neuron 82, 1205-1208 (2014). [PubMed: 24945765]

2. Gooch CL, Pracht E \& Borenstein AR The burden of neurological disease in the United States: A summary report and call to action. Ann. Neurol. 81, 479-484 (2017). [PubMed: 28198092]

3. Abbott NJ, Rönnbäck L \& Hansson E Astrocyte-endothelial interactions at the blood-brain barrier. Nat. Rev. Neurosci. 7, 41-53 (2006). [PubMed: 16371949]

4. Banks WA From blood-brain barrier to blood-brain interface: new opportunities for CNS drug delivery. Nat. Rev. Drug Discov. 15, 275-292 (2016). [PubMed: 26794270]

5. Cho C-F The Blood-Brain Barrier: Brain Cancer Therapy Hits a Wall. Oncol. Times 40, 1 (2018).

6. Cho C-F et al. Blood-brain-barrier spheroids as an in vitro screening platform for brain-penetrating agents. Nat. Commun. 8, 15623 (2017). [PubMed: 28585535] 
7. Urich E et al. Multicellular self-assembled spheroidal model of the blood brain barrier. Sci. Rep. 3, 1500 (2013). [PubMed: 23511305]

8. Wolburg H \& Lippoldt A Tight junctions of the blood-brain barrier: development, composition and regulation. Vascul. Pharmacol. 38, 323-337 (2002). [PubMed: 12529927]

9. Begley DJ \& Brightman MW Structural and functional aspects of the blood-brain barrier. Prog. Drug Res. Fortschritte Arzneimittelforschung Progres Rech. Pharm. 61, 39-78 (2003).

10. Zlokovic BV The blood-brain barrier in health and chronic neurodegenerative disorders. Neuron 57, 178-201 (2008). [PubMed: 18215617]

11. Hervé F, Ghinea N \& Scherrmann J-M CNS delivery via adsorptive transcytosis. AAPS J. 10, 455472 (2008). [PubMed: 18726697]

12. null Schinkel. P-Glycoprotein, a gatekeeper in the blood-brain barrier. Adv. Drug Deliv. Rev. 36, 179-194 (1999). [PubMed: 10837715]

13. Rodriguez A, Tatter SB \& Debinski W Neurosurgical Techniques for Disruption of the BloodBrain Barrier for Glioblastoma Treatment. Pharmaceutics 7, 175-187 (2015). [PubMed: 26247958]

14. Aryal M, Arvanitis CD, Alexander PM \& McDannold N Ultrasound-mediated blood-brain barrier disruption for targeted drug delivery in the central nervous system. Adv. Drug Deliv. Rev. 72, 94109 (2014). [PubMed: 24462453]

15. Demeule $\mathrm{M}$ et al. Involvement of the low-density lipoprotein receptor-related protein in the transcytosis of the brain delivery vector angiopep-2. J. Neurochem. 106, 1534-1544 (2008). [PubMed: 18489712]

16. Stalmans S et al. Cell-Penetrating Peptides Selectively Cross the Blood-Brain Barrier In Vivo. PloS One 10, e0139652 (2015).

17. Zhang Y-Y, Liu H, Summerfield SG, Luscombe CN \& Sahi J Integrating in Silico and in Vitro Approaches To Predict Drug Accessibility to the Central Nervous System. Mol. Pharm. 13, 1540 1550 (2016). [PubMed: 27015243]

18. Bowman PD, Ennis SR, Rarey KE, Betz AL \& Goldstein GW Brain microvessel endothelial cells in tissue culture: a model for study of blood-brain barrier permeability. Ann. Neurol. 14, 396-402 (1983). [PubMed: 6638956]

19. Naik P \& Cucullo L In vitro blood-brain barrier models: current and perspective technologies. J. Pharm. Sci. 101, 1337-1354 (2012). [PubMed: 22213383]

20. Hatherell K, Couraud P-O, Romero IA, Weksler B \& Pilkington GJ Development of a threedimensional, all-human in vitro model of the blood-brain barrier using mono-, co-, and tricultivation Transwell models. J. Neurosci. Methods 199, 223-229 (2011). [PubMed: 21609734]

21. Cecchelli R et al. A Stable and Reproducible Human Blood-Brain Barrier Model Derived from Hematopoietic Stem Cells. PLoS ONE 9, e99733 (2014).

22. Ribecco-Lutkiewicz M et al. A novel human induced pluripotent stem cell blood-brain barrier model: Applicability to study antibody-triggered receptor-mediated transcytosis. Sci. Rep. 8, (2018).

23. Holman DW, Klein RS \& Ransohoff RM The blood-brain barrier, chemokines and multiple sclerosis. Biochim. Biophys. Acta BBA - Mol. Basis Dis. 1812, 220-230 (2011).

24. Hudecz Diána, Rocks Louise, Fitzpatrick Laurence W., Herda Luciana-Maria\& Dawson Kenneth A. Reproducibility in biological models of the blood-brain barrier. Eur. J. Nanomedicine 6, 185 (2014).

25. Griep LM et al. BBB on chip: microfluidic platform to mechanically and biochemically modulate blood-brain barrier function. Biomed. Microdevices 15, 145-150 (2013). [PubMed: 22955726]

26. Booth R \& Kim H Characterization of a microfluidic in vitro model of the blood-brain barrier ( $\mu$ BBB). Lab. Chip 12, 1784-1792 (2012). [PubMed: 22422217]

27. Cecchelli R et al. Modelling of the blood-brain barrier in drug discovery and development. Nat. Rev. Drug Discov. 6, 650-661 (2007). [PubMed: 17667956]

28. Fadzen CM et al. Perfluoroarene-Based Peptide Macrocycles to Enhance Penetration Across the Blood-Brain Barrier. J. Am. Chem. Soc. 139, 15628-15631 (2017). [PubMed: 28992407] 
29. Demeule $M$ et al. Identification and design of peptides as a new drug delivery system for the brain. J. Pharmacol. Exp. Ther. 324, 1064-1072 (2008). [PubMed: 18156463]

30. Maira S-M et al. Identification and characterization of NVP-BKM120, an orally available pan-class I PI3-kinase inhibitor. Mol. Cancer Ther. 11, 317-328 (2012). [PubMed: 22188813]

31. Zou L-L, Ma J-L, Wang T, Yang T-B \& Liu C-B Cell-penetrating Peptide-mediated therapeutic molecule delivery into the central nervous system. Curr. Neuropharmacol. 11, 197-208 (2013). [PubMed: 23997754]

32. Mijalis AJ et al. A fully automated flow-based approach for accelerated peptide synthesis. Nat. Chem. Biol. 13, 464-466 (2017). [PubMed: 28244989]

33. Liu X et al. Molecular imaging of drug transit through the blood-brain barrier with MALDI mass spectrometry imaging. Sci. Rep. 3, 2859 (2013). [PubMed: 24091529]

34. Mittapalli RK, Vaidhyanathan S, Dudek AZ \& Elmquist WF Mechanisms limiting distribution of the threonine-protein kinase $\mathrm{B}-\mathrm{RaF}(\mathrm{V} 600 \mathrm{E})$ inhibitor dabrafenib to the brain: implications for the treatment of melanoma brain metastases. J. Pharmacol. Exp. Ther. 344, 655-664 (2013). [PubMed: 23249624] 


\section{BOX 1|}

\section{HAEMATOXYLIN AND EOSIN STAINING}

1. Immerse slides into $95 \%(\mathrm{v} / \mathrm{v})$ ethanol for 5 minutes.

2. Rinse slides under running tap water for approximately 5 minutes.

3. Dip slides into distilled water for 5 minutes.

4. Immerse slides into haematoxylin for 5 minutes.

5. Rinse slides gently under running tap water for $2-3$ minutes.

6. Immerse slides into tap water for 5 minutes

7. Immerse slides into distilled water for 2 minutes.

8. Dip slides 3-5x into 95\% (v/v) ethanol.

9. Dip slides 1-2x into eosin solution.

10. Dip slides 10-20x into 95\% (v/v) ethanol.

11. Dip slides 10-20x into $100 \%$ (v/v) ethanol. Immerse slides 10-20x into xylene.

12. Mount cover glass onto the sections with mounting medium.

END BOX 


\section{BOX 2|}

\section{TETRAMETHYLRHODAMINE-LABELED ANGIOPEP-2}

Given that the goal is to verify active transport across the BBB, the exact choice of fluorophore and linker are not critical to the success of the experiment. However, for the experiments in Figure 4, the fluorophore was 5-TAMRA and the linker was an amide to the N-terminus. Multiple options exist for obtaining fluorophore-labeled peptides to validate the properties of the BBB organoids

5-TAMRA-labeled peptides may be ordered from a commercial vendor or a core facility. From N- to C- terminus, the peptide sequence of TAMRA-angiopep- 2 is TAMRA-TFFYGGSRGKRNNFKTEEY. The control scrambled-angiopep sequence is TAMRA-GNYTSRFEREYGKFNKFGT.

A. If the user has experience with solid-phase peptide synthesis, the peptides may be prepared through standard solid-phase peptide synthesis using Fmoc chemistry. For the experiments in Figure 4, the peptides were prepared by automated flow peptide synthesis, ${ }^{32}$ and 5-TAMRA carboxylic acid was coupled to the $\mathrm{N}$-terminus of the resin-bound peptide using standard active ester chemistry. After cleavage, the peptides were purified by reversed-phase high-performance liquid chromatography. The purity and identity of the final dye-labeled peptides was confirmed by liquid chromatography massspectrometry. 


\section{a) HBMEC}
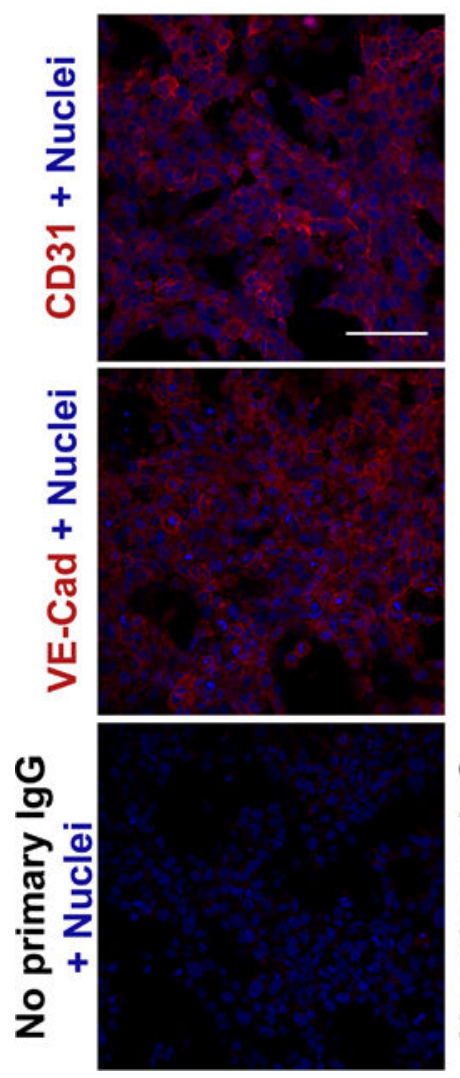

\section{b) HCMEC/D3}
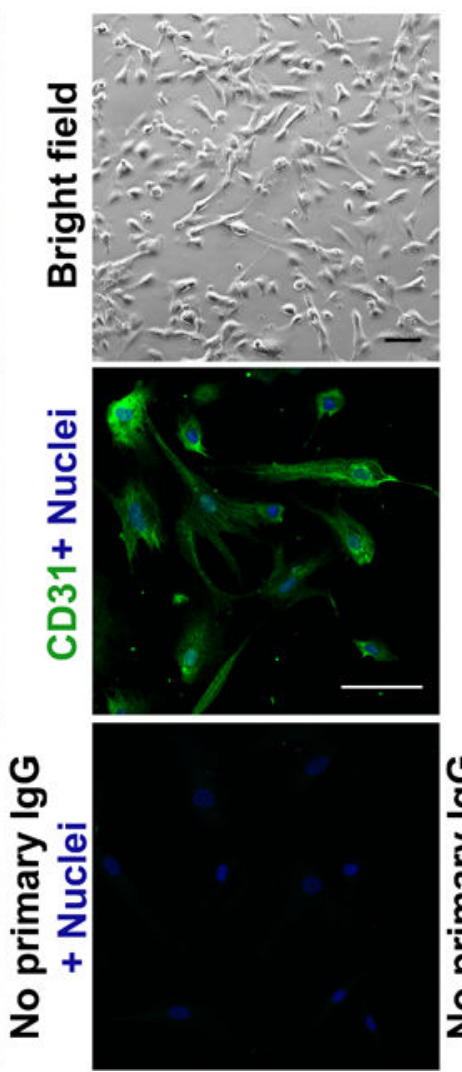

c) HBVP
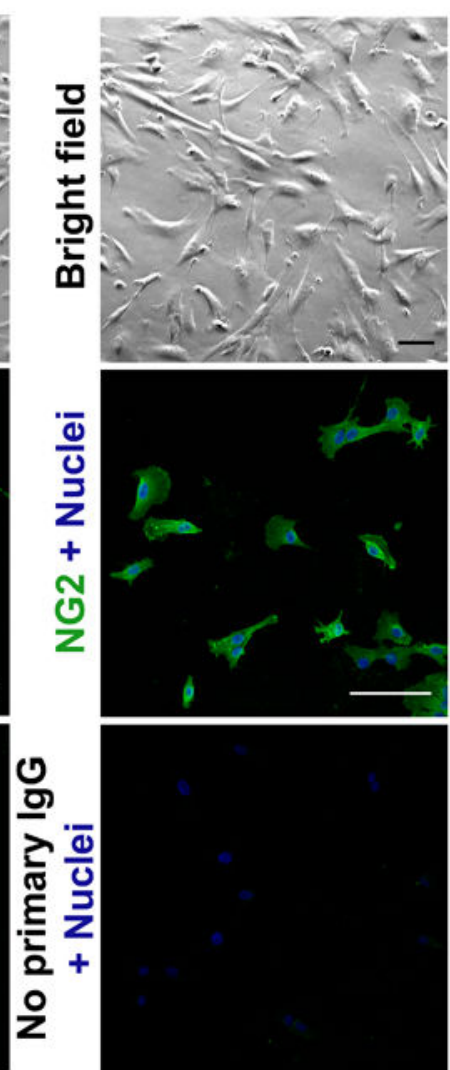

d) Astrocytes
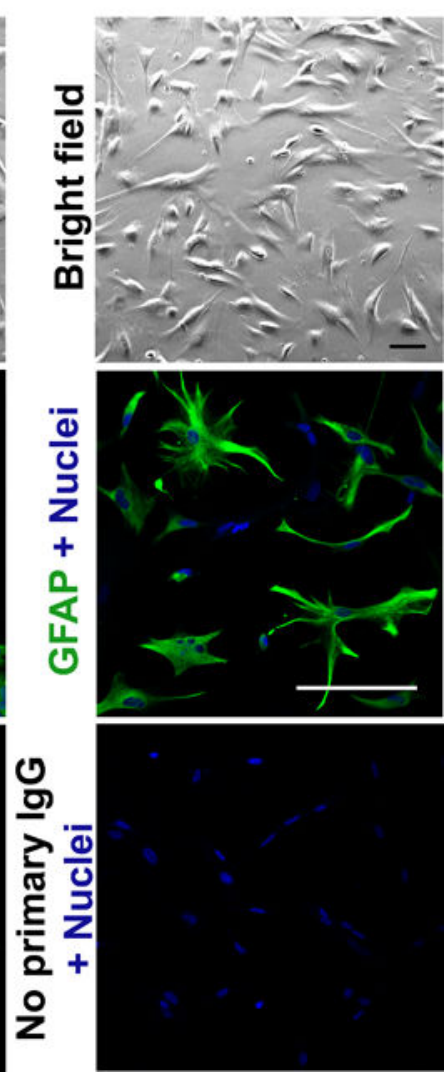

Figure 1|. Characterization of each cell type used for establishing BBB organoids.

a) Fluorescence images showing the expression of CD31 (Abcam, dilution: 1:100) and VECadherin (Cell Signaling Technology, dilution: 1:100) (endothelial cell markers) in primary human brain microvascular endothelial cells (HBMEC) (ScienCell). b) (Top) Bright field image showing the morphology of immortalized human cerebral microvascular endothelial cells (hCMEC/D3) (Cedarlane), and (middle) fluorescence image showing the expression of CD31. c) (Top) Bright field image of primary human brain vascular pericytes (ScienCell) and (middle) fluorescence image showing the expression of the pericyte marker NG2 (Millipore, dilution: 1:100). d) (Top) Bright field image of primary astrocytes (Lonza) and (middle) fluorescence image showing the expression of astrocyte marker GFAP (SigmaAldrich, dilution: 1:100). (b-d, bottom panels) Cells incubated with secondary IgG only (no primary $\operatorname{IgG}$ ) were used as negative controls. Nuclei of cells were stained with the Hoechst dye (blue). Secondary antibody anti-rabbit Alexa Fluor 546 (red) or anti-mouse Alexa Fluor 488 (green) was used. Scale bar: $100 \mu \mathrm{m}$ (Bright field: 20x objective; confocal: 40x objective). 
a)

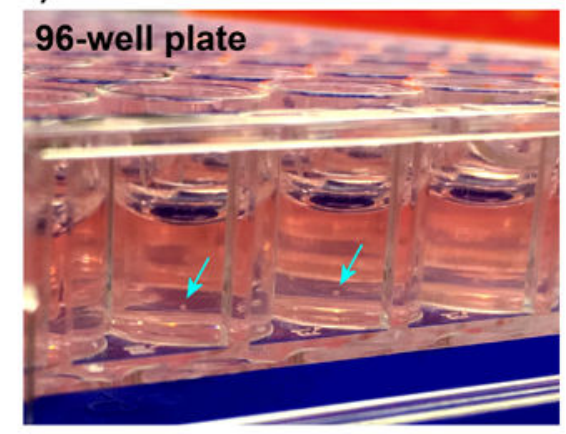

d)

\section{Tight junctions (ZO-1) Secondary IgG only}

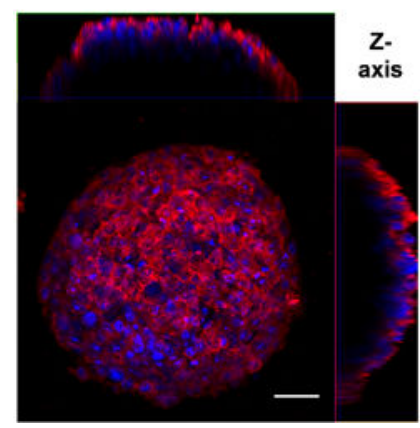

D)
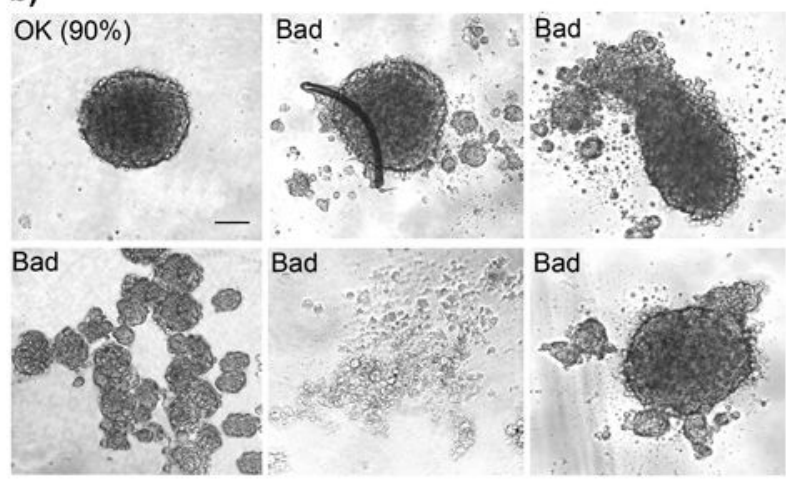

c)

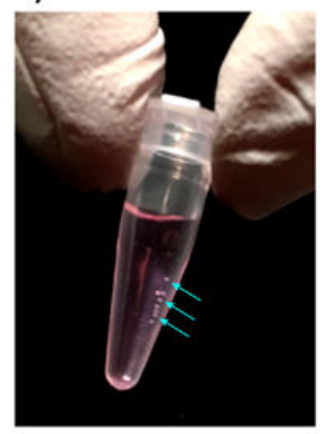

e)

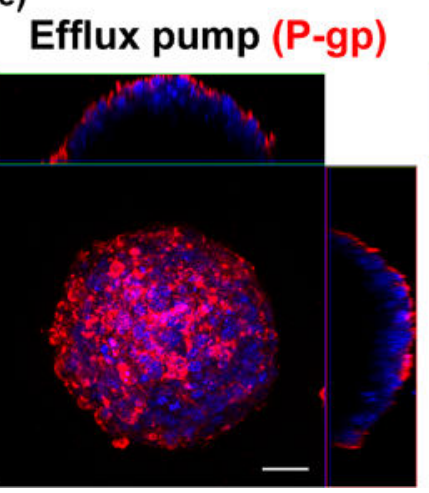

\section{Secondary IgG only}

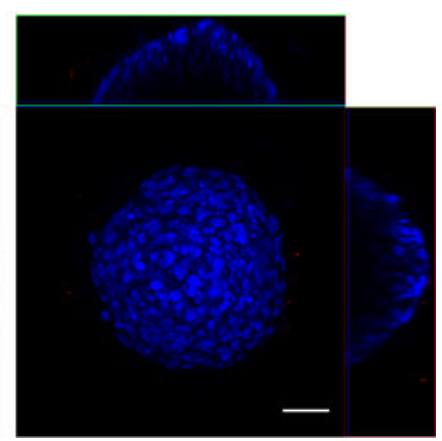

Figure 2|. Culture, collection and assessment of BBB organoids.

a) Formation of multicellular BBB organoids in a 96-well plate by co-culturing hCMEC/D3

(Cedarlane), pericytes (ScienCell) and astrocytes (Lonza) in BBB working medium.

Samples of each cell type were validated by immunofluorescence staining prior to coculturing, as shown in figure 1 . Wells are coated with $1 \%(\mathrm{w} / \mathrm{v})$ agarose to form a concave low-attachment surface. The organoids assemble in the center of each well (blue arrows). b) Representative bright field images showing examples of acceptable and unacceptable organoids on the basis of their physical appearance. In our experiments, out of a total of 1,056 organoids, 950 were considered acceptable, yielding an average success rate of $90 \%$ (cell numbers are pooled from 11 independent organoid cultures). Scale bar: $100 \mu \mathrm{m}(10 \mathrm{x}$ objective). c) Organoids (indicated by cyan arrows) are collected in a microcentrifuge (0.2$\mathrm{mL}$ PCR) tube in BBB working medium. (d) Fluorescence image showing the expression of tight junction marker (ZO-1; red) (Life Technologies, dilution: 1:100) on the organoid surface. (e) Fluorescence image showing the expression of P-glycoprotein (P-gp; red) efflux pump (Abcam, dilution: 1:100) on the organoid surface. Nuclei of organoids are stained with Hoechst dye (blue). As a negative control, organoids are stained with secondary antibody anti-rabbit Alexa Fluor 546 (red) or anti-mouse Alexa Fluor 488 (green) only (no primary antibody). Scale bar: $50 \mu \mathrm{m}$ (40x objective). 
a)

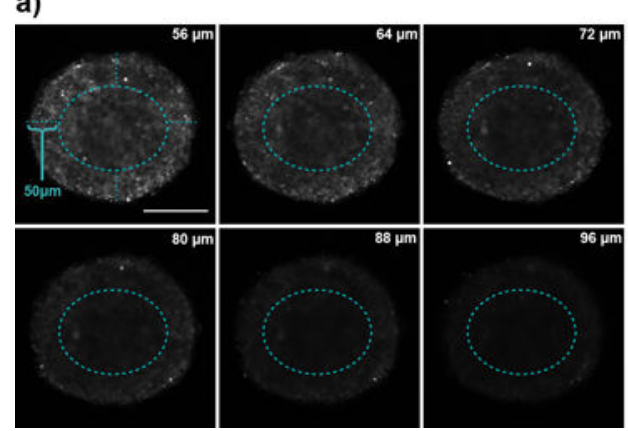

b)

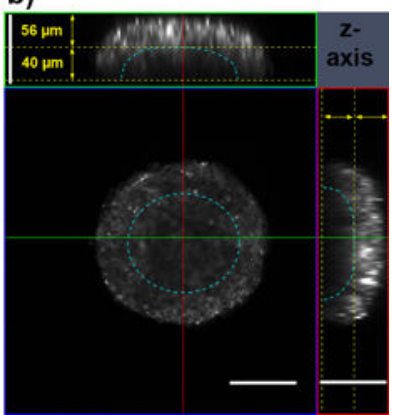

Figure 3|. Quantification of average fluorescence intensity in a BBB organoid.

a) Confocal fluorescence z-stack images of a BBB organoid (at a depth between 56-96 $\mu \mathrm{m}$ ), showing the accumulation of a known BBB-permeable compound, angiopep- $2^{1,2}$ (5 $\mu \mathrm{M}$ concentration, incubated for $5 \mathrm{~h}$ at $37^{\circ} \mathrm{C}$ ) within the organoid core. The core area $50 \mu \mathrm{m}$ from the organoid surface (blue dotted circle) is defined as the region of interest.

Permeability of the compound is determined by measuring the total MFI within the core area of these optical sections. b) Orthogonal view of a organoid (from panel a), showing the core region to be quantified (blue dotted lines). Within the z-direction, quantification is performed at $56 \mu \mathrm{m}$ depth from the surface up to $96 \mu \mathrm{m}$ depth (yellow dotted lines and arrows). Scale bar: $100 \mu \mathrm{m}$ (20x objective). 
a)
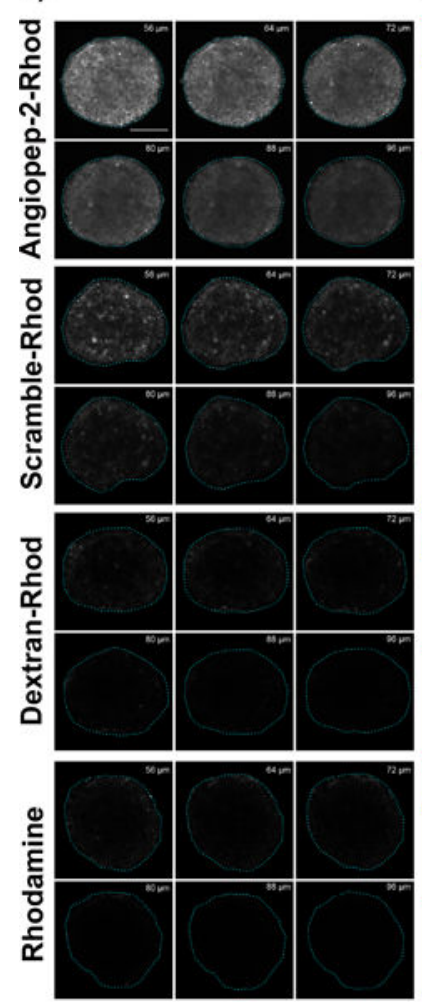

b)
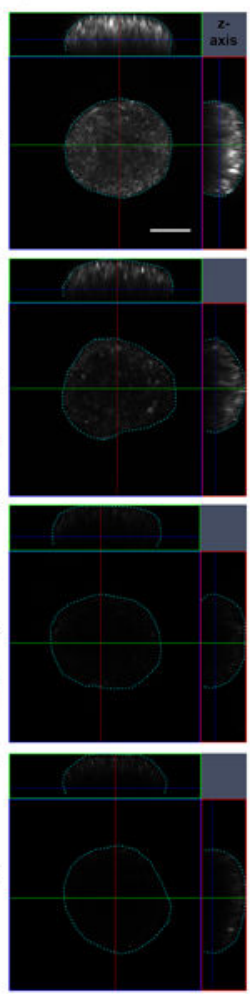

c)

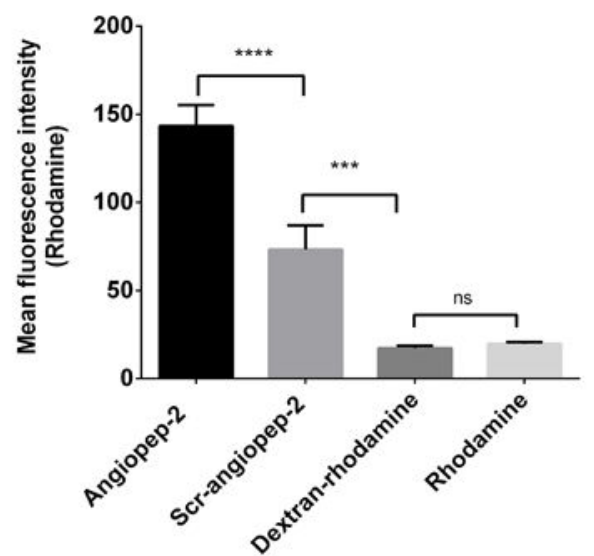

d)

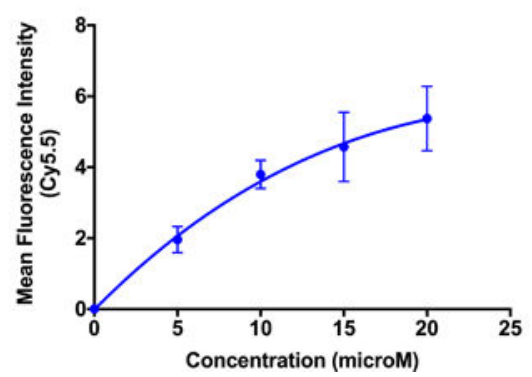

e)

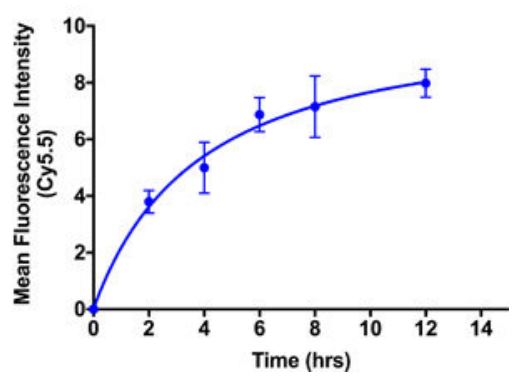

Figure 4|. Analysis of angiopep-2 permeability using the BBB organoid platform by confocal microscopy.

a) Confocal fluorescence z-stack images showing the transport of rhodamine-labeled angiopep-2 (2711.6 Da), rhodamine-scramble peptide (2711.6 Da), dextran-rhodamine (4400 Da) and unconjugated rhodamine dye (380.8 Da) into the BBB organoids. Organoids were established using hCMEC/D3, pericytes and astrocytes, and then, incubated with each compound ( $10 \mu \mathrm{M}$ concentration) at $37^{\circ} \mathrm{C}$ for $5 \mathrm{hrs}$. Each organoid was imaged by confocal fluorescence microscopy (using a 20x objective), and the mean fluorescence intensity in the organoid core was quantified using ImageJ. b) Orthogonal view of the organoids showing the distribution of the compounds. White dotted lines indicate organoid border. Scale bar: $100 \mu \mathrm{m}$. c) Bar graph displaying the quantification of the total mean fluorescence intensity of the optical sections (from a)). Statistical analysis was performed using One-way ANOVA and Tukey's multiple comparisons test. Graph depicts the sum of the mean fluorescence intensity of the core area from optical sections at a depth of $56-96 \mu \mathrm{m}$ with standard deviation error bars $\left(* * * * p<0.0001 ; * * * p<0.001 ; n_{\text {organoids }}=5\right)$. d) Graph depicting the transport of $\operatorname{Bip}(1)$ in a concentration-dependent manner after $2 \mathrm{hrs}$ of incubation $\left(\mathrm{n}_{\text {spheroid }}=\right.$ 5-7). e) Time course analysis of the transport of Cy5-Bip(1) into the BBB organoid over 26 hours (at $10 \mu \mathrm{M}$ concentration) $\left(\mathrm{n}_{\text {spheroid }}=4-6\right)$. The graphs display mean Cy5.5 fluorescence intensity quantified at $88 \mu \mathrm{m}$ depth from the surface of each spheroid with SD error bars. 
a)

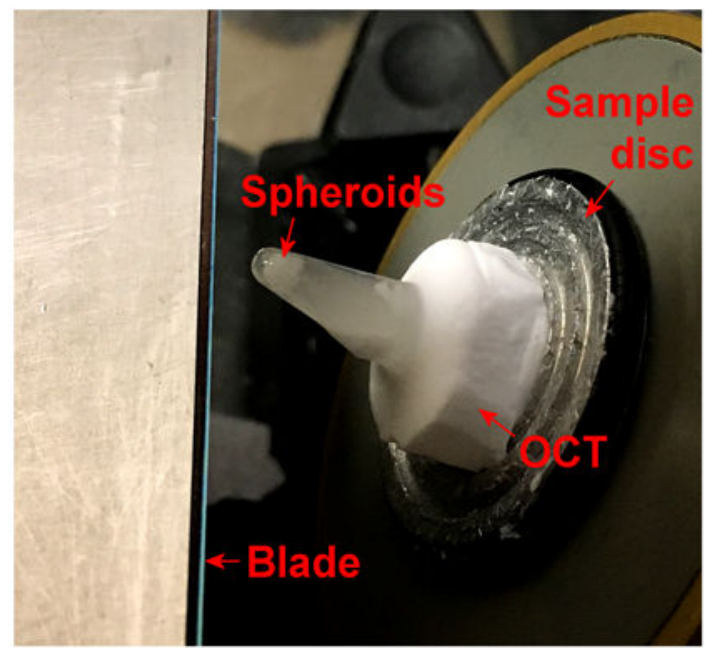

c)

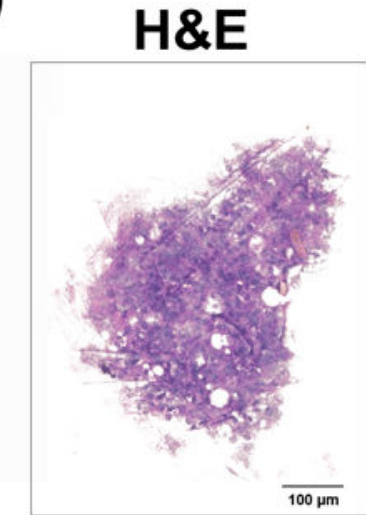

b)

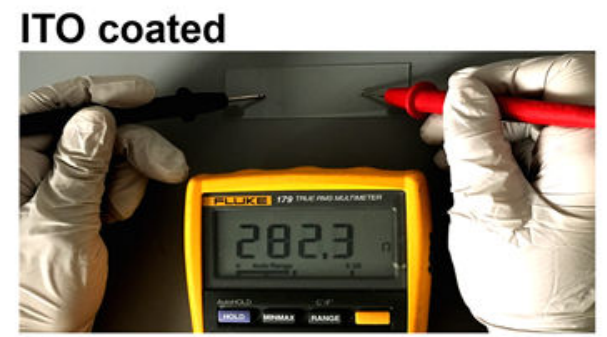

ITO uncoated

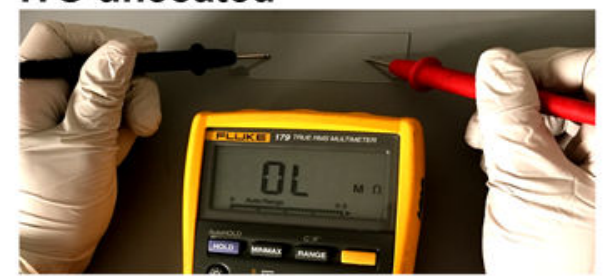

Pixel
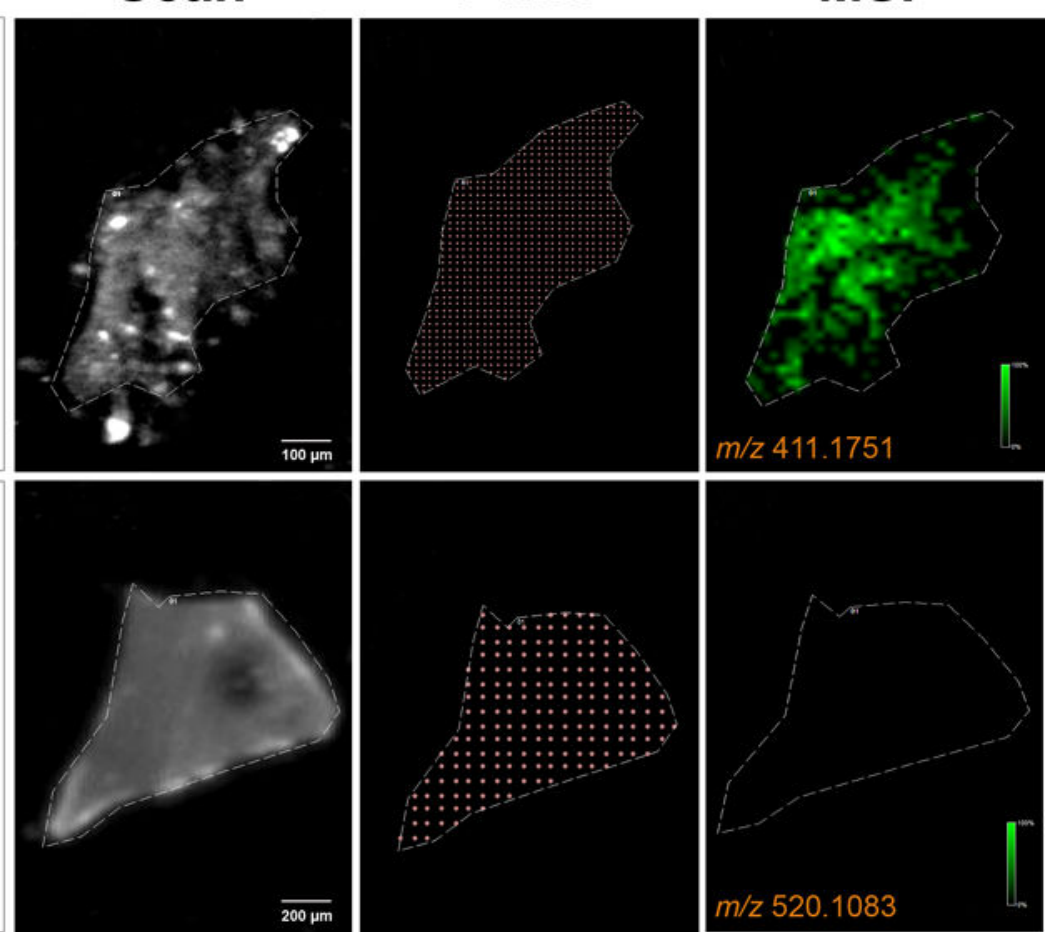

$m / Z 411.175$

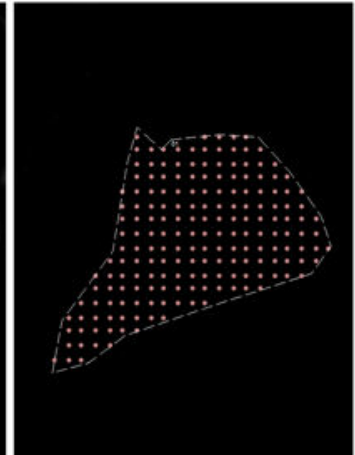

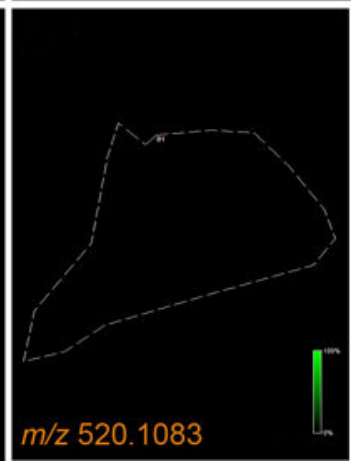

Figure 5|. Setup and analysis of BKM120 and dabrafenib permeability by MALDI-MSI.

a) Setup of the cryostat for sectioning of BBB organoids. Organoids were collected at the bottom of a PCR tube and snap frozen in a dry ice/ethanol bath. The cap of the tube was then mounted onto the sample disc. b) Conductivity was measured on both sides of an indium tin oxide (ITO) coated glass slide with a voltage-meter to determine the correct ITO coated side for mounting of tissue sections. ITO coating resulted in a measurable electrical resistance on the surface of the slide, while the uncoated side was non-conductive. For MSI, cryo-sectioned tissues must be mounted onto the ITO-coated side. c) H\&E stained images of 
the cryo-sections from each group confirmed the presence of BBB organoid tissues ( $\mathrm{n}_{\text {organoid }}$ =150). Serial sections were used for MSI. 'Scan' images were used to identify the position of organoid sections on the ITO slide. 'Pixel' images show the pixel distribution within the selected scanned region (spatial resolution of $30 \mu \mathrm{m}$ ). MALDI MSI ion images reveal the distribution/level of both drugs in the organoid sections, showing a high level of BKM120 accumulation within the organoids (in green $\mathrm{m} / \mathrm{z} 411.1751 \pm 0.001$ ). Dabrafenib was not detected within the organoids $(\mathrm{m} / \mathrm{z} 520.1083 \pm 0.001)$. Scale bars for BKM image: $100 \mu \mathrm{m}$, Scale bar for Dabrafenib image: $200 \mu \mathrm{m}$. Images from panel (c) are adapted from Cho et al., Nature Communications (2017). ${ }^{6}$ 


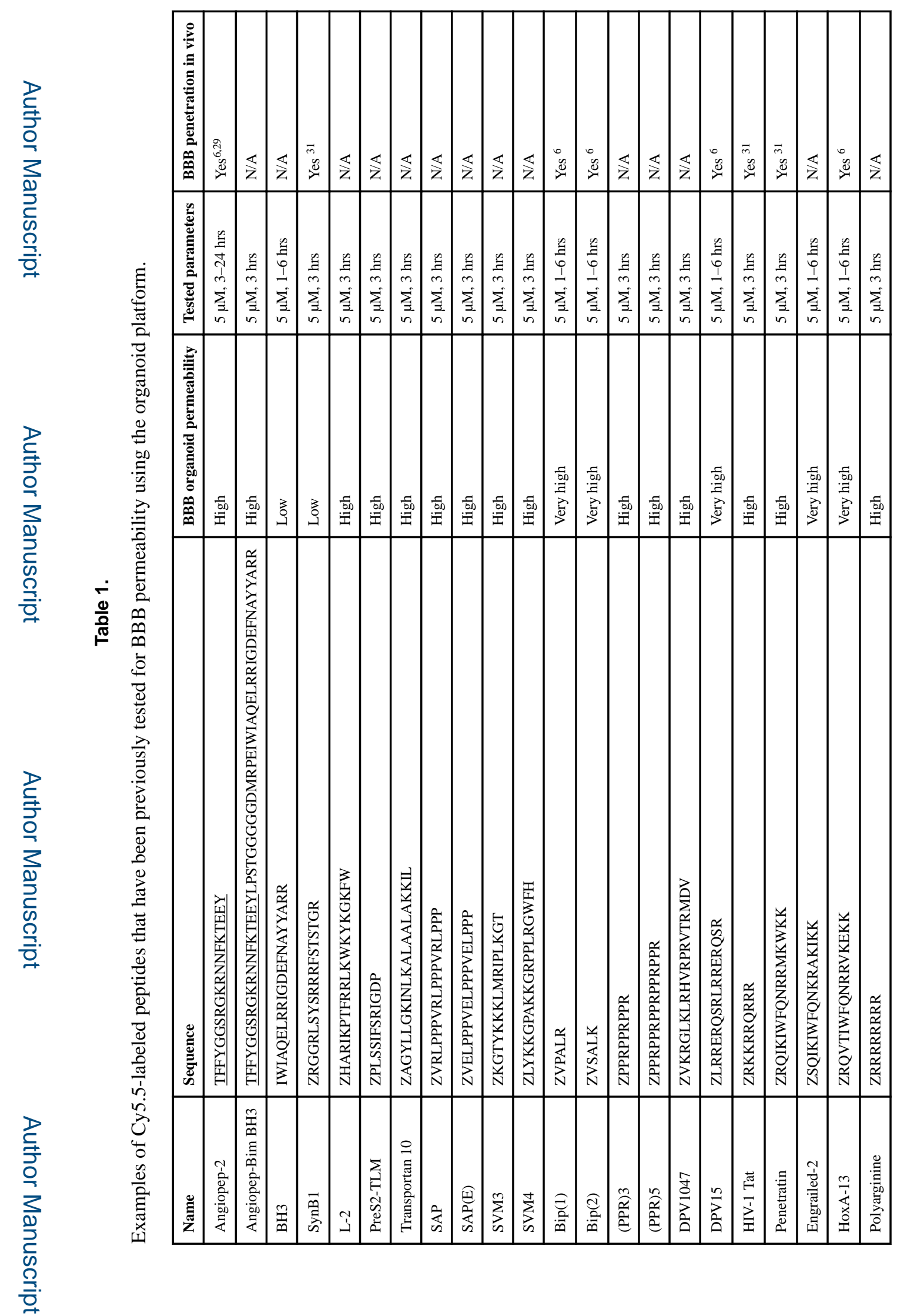

Nat Protoc. Author manuscript; available in PMC 2019 December 01. 
Table 2.

The $\mathrm{m} / \mathrm{z}$ ratio of small molecule compounds that we have previously analyzed.

\begin{tabular}{|l|l|}
\hline Compound & $\boldsymbol{m} / \boldsymbol{z}[\mathbf{M}+\mathbf{H}]$ \\
\hline BKM120 & 411.1751 \\
\hline Dabrafenib & 520.1083 \\
\hline & \\
\hline & \\
\hline
\end{tabular}


TABLE 3|

Troubleshooting table

\begin{tabular}{|c|c|c|c|}
\hline Step & Problem & Reason & Solution \\
\hline 1 & $\begin{array}{l}\text { Debris present within formed organoids } \\
\text { (see Fig. 2c) }\end{array}$ & $\begin{array}{l}\text { Medium contains precipitates or } \\
\text { crystals from FBS }\end{array}$ & $\begin{array}{l}\text { Filter supernatant through a } 0.8 \mu \mathrm{m} \text { sterile } \\
\text { filter before adding to culture medium. }\end{array}$ \\
\hline 7 & $\begin{array}{l}\text { Uneven agarose surface can lead to } \\
\text { unformed or deformed organoids }\end{array}$ & Uneven or damaged agarose surface & $\begin{array}{l}\text { Ensure that } 1 \%(\mathrm{w} / \mathrm{v}) \text { agarose solution is fully } \\
\text { dissolved during the heating process (no } \\
\text { precipitates). Work quickly to dispense the } \\
\text { agarose solution into each well of a } 96 \text {-well } \\
\text { plate while it is hot to prevent solidification as } \\
\text { the solution cools. Even partial gelling can } \\
\text { cause an uneven surface to form. Leave the } \\
\text { agarose to cool and solidify properly at room } \\
\text { temperature for at least } 15 \text { minutes and avoid } \\
\text { moving of the plate. }\end{array}$ \\
\hline 14 & Formation of multiple small organoids & $\begin{array}{l}\text { Cell clumping (not in cell single cell } \\
\text { form) after trypsinization or before } \\
\text { cell seeding. }\end{array}$ & $\begin{array}{l}\text { Ensure that resuspended trypsinized cells are } \\
\text { in single-cell form. Count and seed cells } \\
\text { immediately (within } 10-15 \text { minutes) after } \\
\text { trypsinization. Assess that single cells are } \\
\text { being seeded by investigating each well under } \\
\text { an inverted microscope after each seeding } \\
\text { step. }\end{array}$ \\
\hline $\begin{array}{l}15 \text { A iii, } \\
\text { B iii, C } \\
\text { i }\end{array}$ & $\begin{array}{l}\text { Test compound precipitates in } \mathrm{BBB} \\
\text { working medium }\end{array}$ & Compound is water-insoluble. & $\begin{array}{l}\text { Dissolve compound of interest first in } \\
\text { Kollisolv }{ }^{\circledR} \text { PEG } 300 \text { (Sigma Aldrich) and } \\
\text { then dilute in BBB working medium or D- } \\
\text { PBS to a final percentage of } 10 \%(\mathrm{v} / \mathrm{v}) \text { PEG } \\
300 \text {. }\end{array}$ \\
\hline $\begin{array}{l}15 \text { A iv, } \\
\text { B iv }\end{array}$ & Organoids merge/clump together & $\begin{array}{l}\text { Organoids are in contact with one } \\
\text { another over an extended period of } \\
\text { time. }\end{array}$ & $\begin{array}{l}\text { Once organoids are pooled together, keep } \\
\text { them under rotation throughout the incubation } \\
\text { and ensure that the organoids are constantly } \\
\text { suspended in the solution. }\end{array}$ \\
\hline $\begin{array}{l}16 \mathrm{~A} \mathrm{v} \\
16 \mathrm{~B} \text { i }\end{array}$ & $\begin{array}{l}\text { Loss of organoids due to them sticking } \\
\text { to the inside wall of a pipette tip }\end{array}$ & $\begin{array}{l}\text { Organoid's propensity to adhere to the } \\
\text { surface of plastic. }\end{array}$ & $\begin{array}{l}\text { Coat pipette tip with PBS containing } 0.1- \\
0.2 \%(\mathrm{v} / \mathrm{v}) \text { Triton X-100. }\end{array}$ \\
\hline
\end{tabular}

